\title{
Oscillations trumped by behavior: a link between sensory and direct electrical stimulation of cortical activity
}

\author{
Adriano B. L. Tort ${ }^{*}$ and Donald B. Katz ${ }^{2}$ \\ Edmond and Lily Safra International Institute of Neuroscience of Natal and Federal University of Rio Grande do Norte, Natal, Rio Grande do Norte, Brazil \\ 2 Department of Psychology/Program of NeuroscienceNolen National Center for Complex Systems, Brandeis University, Waltham, MA, USA \\ ${ }^{*}$ Correspondence: tort@natalneuro.org.br
}

\section{A commentary on}

Behavioral modulation of stimulus-evoked oscillations in barrel cortex of alert rats

by Venkatraman, S., and Carmena, J. M. (2009). Front. Integr. Neurosci. 3:10. doi:10.3389/neuro.07.010.2009.

Behavior is well known to influence spiking responses in both thalamus and cortex to either sensory stimuli or electrical stimulation of afferent nerves (Chapin and Woodward, 1981; Fanselow and Nicolelis, 1999; Fontanini and Katz, 2009). This fact has been particularly well documented in the rodent somatosensory system, where whisking behavior tends to decrease the initial response of thalamic and cortical neurons to activation of the trigeminal nerve, as well as the prolonged suppression of activity that commonly follows this initial response (Fanselow and Nicolelis, 1999).

In a largely unconnected set of experiments, sensory cortices have been shown to exhibit oscillations at particular frequencies following direct or sensory stimulation, as measured in field potentials (Contreras and Steriade, 1996; Muthuswamy et al., 1999). The fact that these phenomena are more commonly observed under systemic anesthesia (e.g., Derdikman et al., 2003) than in awake animals (but see Kay, 2005; Tort et al., 2010), makes it tempting to conclude, in light of the above-described work, that awake behavior might suppress both neuronal responses and oscillatory activity. Now, in Frontiers, Venkatraman and Carmena (2009) employ direct cortical recording and stimulation techniques that they have recently developed (Venkatraman et al., 2009) to provide evidence that this is indeed the case. These findings also suggest, for the first time, common cellular and network mechanisms underlying the cortical response to direct electrical activation and peripheral sensory stimulation.
Working with awake, whisking rats, Venkatraman and Carmena (2009) start with the observation that direct electrical microstimulation of the barrel cortex induces oscillations in the $15-18 \mathrm{~Hz}$ range, but that this induction is far from $100 \%$ reliable. An important factor underlying this variability, they suggest, is the behavioral state of the rat, as relates to whisking behavior: prominent oscillations are induced when stimulation is delivered to an animal exhibiting quiet immobility, but much less rhythmic activity is induced during active whisking. In fact, the authors show a direct, inverse correlation between the amplitude of the stimulation-induced oscillations and the strength of whisking during stimulation; they also demonstrate that this inverse relation with behavior occurs very precisely in time, and rule out potentially confounding variables such as the level of attention. These results effectively bring together the previous findings described above, showing that motor behavior similarly affects induced spiking responses and network oscillations. In fact, Vekatraman and Carmena make this similarity explicit, showing that the strength of spike inhibition following the immediate burst of stimulation-induced spikes also exhibits a negative dependence on whisking behavior.

Venkatraman and Carmena go on to simulate a feasible biophysical substrate for the observed dynamics. Making clever use of previous modeling work (Suffczynski et al., 2004), they show how strong feedforward inhibition (possibly involving $\mathrm{GABA}_{\mathrm{B}}$ ) could play a chief role in inducing thalamic neurons to burst (this thalamic bursting would be transmitted to cortical neurons as the observed oscillations), and suggest that the deinactivation of thalamic $I_{T}$ currents promoted by strong hyperpolarization is crucial to rebound excitation and beginning of bursting activity in these cells $\left(I_{T}\right.$ currents are inactivated during excitation, and are set to deinactivate during prolonged inhibition; for reviews on this mechanism of bursting generation, see Sherman, 2001; Steriade, 2005; Huguenard and McCormick, 2007). According to this model, whisking would likely provide a greater level of excitation to thalamic neurons (e.g., by increased sensory inputs or by a greater action of neuromodulators such as acetylcholine), which would counteract hyperpolarization, keeping $I_{T}$ inactivated and decreasing the likelihood of bursts and oscillations. Responses are thus governed by an appealing balance between corticothalamic feedforward inhibition and behaviorally induced thalamic excitation. The authors' demonstration that they could induce cortical oscillations even in the presence of active whisking behavior by increasing the intensity of the stimulation is compatible with their model, assuming that the generation of oscillations is dependent on the number of cortical neurons recruited.

The functional consequences of these observations remain to be explored. It has been suggested that thalamocortical network settings in "quiet" animals are optimized for stimulus detection (such that spiking responses are large and bursting). During active exploration, neurons are tonically active, such that they are better prepared to perform a more fine-grained analysis of stimulus dynamics (Nicolelis and Fanselow, 2002). It may well be that the differences in oscillatory power observed in response to cortical stimulation are a slightly more direct assay of these differences in network settings, rather than being the purpose of the network settings per se.

This is a particularly pertinent issue given that multiple distinct but overlapping rhythms can be observed in cortical circuits (Tort et al., 2010). That is, the function of the induced rhythm may be a subtle function of precisely what rhythm is induced. The authors specifically suggest that their observed oscillations more closely resemble the spindles observed under ketamine-xylazine anesthesia than 
the spontaneous spike-and-wave shaped " $\mu$ " rhythm that induces whisker twitching, and show that a blockade of GABA $_{A}$ can conceivably participate in making their thalamocortical network model transition from one of these rhythms to the other. With this combination of behavior, oscillation induction, and modeling, Venkatraman and Carmena are therefore poised to integrate a disparate body of results, and provide us with a uniquely wholistic explanation of thalamocortical function in awake rats.

\section{REFERENCES}

Chapin, J. K., and Woodward, D. J. (1981). Modulation of sensory responsiveness of single somatosensory cortical cells during movement and arousal behaviors. Exp. Neurol. 72, 164-178.

Contreras, D., and Steriade, M. (1996). Spindle oscillation in cats: the role of corticothalamic feedback in a thalamically generated rhythm. J. Physiol. (Lond.) 490, 159-179.

Derdikman, D., Hildesheim, R., Ahissar, E., Arieli, A., and Grinvald, A. (2003). Imaging spatiotemporal dynamics of surround inhibition in the barrels somatosensory cortex. J. Neurosci. 23, 3100-3105.
Fanselow, E. E., and Nicolelis, M. A. (1999). Behavioral modulation of tactile responses in the rat somatosensory system. J. Neurosci. 19, 7603-7616.

Fontanini, A., and Katz, D. B. (2009). Behavioral modulation of gustatory cortical activity. Ann. N. Y. Acad. Sci. 1170, 403-406.

Huguenard, J. R., and McCormick, D.A. (2007). Thalamic synchrony and dynamic regulation of global forebrain oscillations. Trends Neurosci. 30, 350-356.

Kay, L. M. (2005). Theta oscillations and sensorimotor performance. Proc. Natl. Acad. Sci. U.S.A. 102, 3863-3868.

Muthuswamy, J., Tran, P., Rangarajan, R., Lenz, F. A. Hanley, D. F., and Thakor, N.V. (1999). Somatosensory stimulus entrains spindle oscillations in the thalamic VPL nucleus in barbiturate anesthetized rats. Neurosci. Lett. 262, 191-194.

Nicolelis, M.A., andFanselow,E.E. (2002). Thalamocortical optimization of tactile processing according to behavioral state. Nat. Neurosci. 5, 517-523.

Sherman, S. M. (2001). Tonic and burst firing: dual modes of thalamocortical relay. Trends Neurosci. 24, 122-126.

Steriade, M. (2005). Sleep, epilepsy and thalamic reticular inhibitory neurons. Trends Neurosci. 28, 317-324.

Suffczynski, P., Kalitzin, S., and Lopes Da Silva, F. H. (2004). Dynamics of non-convulsive epileptic phenomena modeled by a bistable neuronal network Neuroscience 126, 467-484.

Tort,A.B.,Fontanini,A., Kramer,M.A.,Jones-Lush,L., Kopell, N., and Katz,D.B.(2010).Cortical networks produce three distinct 7-12 Hzrhythms during single sensory responses in the awake rat. J. Neurosci. 30, 4315-4324.

Venkatraman, S., and Carmena, J. M. (2009). Behavioral modulation of stimulus-evoked oscillations in barrel cortex of alert rats. Front. Integr. Neurosci. 3:10. doi: 10.3389/neuro.07.010.2009.

Venkatraman, S., Elkabany, K., Long, J. D., Yao, Y., and Carmena, J. M. (2009). A system for neural recording and closed-loop intracortical microstimulation in awake rodents. IEEE Trans. Biomed. Eng. 56, 15-22.

Conflict of Interest Statement: The authors declare that the research was conducted in the absence of any commercial or financial relationships that could be construed as a potential conflict of interest.

Received: 23 February 2010; paper pending published: 22 March 2010; accepted: 27 May 2010; published online: 15 September 2010.

Citation: Tort ABL and Katz DB (2010) Oscillations trumped by behavior: a link between sensory and direct electrical stimulation of cortical activity. Front. Neurosci. 4:39. doi: 10.3389/fnins.2010.00039

Copyright $(2010$ Tortand Katz. This is an open-access article subject to an exclusive license agreement between the authors and the Frontiers Research Foundation, which permits unrestricted use, distribution, and reproduction in any medium, provided the original authors and source are credited. 